\title{
La valutazione degli aspetti qualitativi dell'assistenza fornita dai servizi psichiatrici e l'integrazione con i dati dei registri e dei sistemi informativi
}

\author{
MIRELLA RUGGERI
}

I dati forniti dai registri dei casi e dai sistemi informativi hanno fornito nell'ultimo ventennio informazioni di primaria importanza per la valutazione dei servizi psichiatrici (Wing \& Fryers, 1976 ten Horn et al., 1986; Tansella, 1989; De Salvia \& Tansella, $1992 \mathrm{a}, \mathrm{b})$. È, tuttavia, emersa sempre più chiaramente la necessità di integrare tali dati tipicamente quantitativi (caratteristiche socio-demografiche degli utenti, incidenza e prevalenza dei disturbi psichici «trattati» nei servizi, numero e caratteristiche dei contatti, ecc.) con una valutazione di tipo qualitativo (sintomi clinici, funzionamento sociale, bisogni assistenziali, ecc.).

Classicamente, la valutazione di un servizio avviene attraverso la valutazione della struttura (edifici, risorse economiche, personale), del processo (le attività svolte dal personale del servizio) e dell'esito dell'intervento. Il peso della componente qualitativa aumenta dalla prima alla terza tappa ed il riconoscimento della sua rilevanza è diventato maggiore nel corso degli anni, man mano che si sono affinate le conoscenze e le riflessioni nell'ambito della psichiatria sociale.

La definizione teorica dell'esito, e di conseguenza la sua misurazione, ha costituito, e costituisce, un problema non facilmente affrontabile. In passato i ricercatori hanno di frequente considerato dati riguardanti la struttura e, soprattutto, il processo dell'intervento (in particolare parametri relativi all'utilizzazione dei servizi quali, ad esempio, il numero di contatti e di ricoveri) come indicatori di esito. In effetti tale confusione nasceva dalla insoddisfazione dei ri-

Indirizzo per la corrispondenza: Dr.ssa Mirella Ruggeri, Servizio di Psicologia Medica, Istituto di Psichiatria, Ospedale Policlinico, 37134 Verona.

Fax $(+39)$ 45-585871. cercatori e dei clinici per i parametri tradizionali di esito, quali la morbilità e la mortalità, ritenuti, soprattutto nel caso di disturbi psichici cronicamente invalidanti, riduttivi e scarsamente sensibili a mutamenti a breve termine. Il presupposto razionale di questa prassi era, da un lato, che l'intensità dell'utilizzo fosse in qualche modo collegata con la gravità del disturbo e, dall'altro, che l'utilizzo fosse in qualche modo correlato con il risultato finale dell'intervento. Oggi sappiamo che esito ed utilizzazione dei servizi non sono necessariamente correlati, né tanto meno coincidono, e che l'utilizzazione dei servizi può dipendere da variabili di natura eterogenea, quali le caratteristiche sociodemografiche dei pazienti o la qualità della relazione del paziente con l'operatore, oltre che da caratteristiche «intrinseche» del servizio stesso.

Quali sono, dunque, le variabili che contribuiscono in maniera determinante all'esito di un intervento psichiatrico e quali sono i principi per una loro corretta misurazione? Allo stato attuale delle conoscenze possono essere individuate alcune linee-guida.

1. La misurazione dell'esito deve essere multidimensionale e riguardare, in particolare, aspetti clinici, sociali e di interazione con i servizi psichiatrici.

La determinazione dell'esito clinico di un intervento include principalmente lo studio della gravità e della variabilità dei sintomi e la loro aggregazione di entità diagnostiche definite. È questo uno degli ambiti in cui la psichiatria epidemiologica ha compiuto maggiori progressi negli ultimi anni, con lo sviluppo di strumenti sofisticati per misurare i sintomi e per porre diagnosi secondo i criteri diagnostici più accreditati (Thompson, 1989).

Di natura eterogenea e più complessa misurazione è l'esito dal punto di vista sociale. Contributi provenienti dall'ambito della psichiatria sociale hanno por- 
tato alla luce con chiarezza la complessità del rapporto fra sintomi clinici e funzionamento sociale dell'individuo (de Jong et al., 1986): l'esito nelle due aree può, infatti, essere sovrapponibile in alcuni casi (ad esempio, miglioramento dei sintomi clinici con miglioramento del funzionamento sociale), o completamente diverso in altri (ad esempio, nessun cambiamento dei sintomi clinici con miglioramento del funzionamento sociale). È stato dimostrato che tale articolazione può dipendere, fra gli altri fattori, anche dalle caratteristiche dell'intervento attuato: ad esempio, è stato dimostrato che la permanenza di pazienti con gravi disturbi psichici nella comunità anziché nell'ospedale psichiatrico determina un miglioramento più marcato delle abilità sociali che dei sintomi clinici (Mignolli et al., 1991).

Fra i parametri correlati al funzionamento sociale dell'individuo, e frequentemente alterati nei pazienti con disturbi psichici gravi, segnaliamo il supporto sociale; la dimostrazione di un miglior esito dal punto di vista del funzionamento sociale nei pazienti con maggior supporto sociale (Brugha, 1990) ha spinto i ricercatori a meglio definire la natura e l'evoluzione della rete dei contatti sociali e a costruire strumenti in grado di misurarne adeguatamente le caratteristiche; questi dati sottolineano, inoltre, l'opportunità che i servizi psichiatrici, nel programmare le strategie terapeutiche da attuare, si pongano anche l'obiettivo di riforzare la rete «naturale» di contatti di ciascun paziente (e non solo, come frequentemente accade, in qualche modo di sostituirla).

Un'altra variabile sta conquistando sempre maggior rilievo come indicatore di esito per i pazienti affetti da gravi disturbi mentali: la qualità della vita, considerata in ambiti differenti, quali l'abitazione, le condizioni economiche, il lavoro, le relazioni sociali, la salute, il tempo libero (Lehman et al., 1990). Alta priorità dovrà essere data nei prossimi anni allo studio del rapporto fra qualità della vita, psicopatologia, funzionamento sociale e stato di salute generale, all'ottenimento di conoscenze scientifiche sulla stabilità della qualità di vita nel tempo ed alla identificazione degli ambiti di vita maggiormente compromessi nei pazienti con gravi disturbi psichici.

Lo studio dell'interazione fra utenti e servizi psichiatrici, infine, con la valutazione di parametri quali il carico familiare (Fadden et al., 1987), i bisogni (Wing, 1990; Thornicroft et al., 1992) e la soddisfazione degli utenti (Ruggeri, 1991), costituisce uno dei filoni maggiormente innovativi nella valutazione dei servizi psichiatrici. La soggettività di questi parametri pone indubbi problemi teorici e metodologici che hanno, per molti anni, rallentato e reso assai complesso il lavoro di ricerca in questo ambito. Va, tuttavia, riconosciuto che proprio nella soggettività di tali valutazioni risiede la loro rilevanza: prenderle in considerazione è, infatti, il solo modo per gettar luce su molteplici dimensioni dell'interazione fra utenti e servizi che sfuggirebbero ad un'osservazione effettuata esclusivamente "attraverso gli occhi» degli operatori o dei ricercatori e che hanno un ruolo di enorme importanze nel determinare lo stile dell'interazione con il servizio e la sua efficacia.

La soddisfazione è il più soggettivo fra i parametri citati; la «praticabilità» e l'utilità di tale misurazione è stata spesso messa in discussione, in quanto venivano espressi dubbi sulla reale capacità dei pazienti psichiatrici di valutare in maniera attendibile la propria esperienza. Questo pregiudizio è stato ampiamente smentito in uno studio sulle aspettative e la soddisfazione dei pazienti, dei loro familiari e degli operatori da noi recentemente effettuato presso il servizio psichiatrico di Verona-Sud (Ruggeri \& Dall'Agnola, 1993; Ruggeri et al., 1993), in cui gli utenti, inclusi i soggetti affetti da gravi disturbi psichici, sono risultati in grado di esprimere un'opinione coerente, stabile e di estremo interesse dal punto di vista valutativo.

2. La misurazione dell'esito deve essere fatta secondo prospettive multiple, secondo, cioè, i punti di vista di soggetti con ruoli diversi e un diverso coinvolgimento nell'assistenza (ad esempio il paziente, $\mathrm{i}$ suoi familiari, il personale medico, il personale paramedico, ecc.). Come già citato al punto 1., è possibile che questi soggetti abbiano punti di vista diversi sull'esito; poiché nessun punto di vista può a priori essere considerato maggiormente attendibile, una corretta valutazione dell'esito dovrà essere effettuata attraverso una misurazione multiassiale e derivare dalla integrazione dei molteplici punti di vista. Alla luce di contributi provenienti da vari ambiti risulta, poi, chiaro che la componente soggettiva, e la possibilità che esistano prospettive diverse, non è una caratteristica di pertinenza esclusiva dei parametri che valutano l'interazione fra utenti e servizi ma un elemento fondamentale anche della valutazione dei sintomi clinici e del funzionamento sociale (Attkisson et al., 1992). Quanto detto indica l'impossibilità, per la maggior parte dei parametri di esito, di una misurazione univoca ed "obiettiva», mette in discussione il ruolo dell'operatore (o del ricercatore) come unico giudice dell'esperienza del paziente ed ulteriormente sottolinea la necessità di una integrazione dei dati hard 
provenienti dai registri dei casi e dai sistemi informativi con i dati soft prodotti da una valutazione di tipo qualitativo.

3. La misurazione dell'esito deve essere sensibile e longitudinale. A tale scopo, dovranno essere avviati studi in grado di individuare l'influenza sull'esito delle caratteristiche dei soggetti (età e sesso possono, ad esempio, grandemente influenzare le prospettive sull'esito desiderabile). Alta priorità dovrà essere data all'effettuazione di studi comparativi (e, possibilmente, controllati) fra interventi diversi, che determinino quali sono gli interventi più efficaci, in quali condizioni, per quali pazienti (a tutt'oggi, ad esempio, non è stata confrontata l'efficacia di molte strategie riabilitative e psicoterapeutiche). Occorrono, inoltre, studi longitudinali che determinino l'efficacia a lungo termine dei vari interventi e monitorizzino l'esito con misurazioni ripetute nel tempo. Importante sarà anche correlare l'efficacia degli interventi con il loro costo.

L'attuazione delle tre linee-guida illustrate richiede una acquisizione metodologica preliminare: lo sviluppo e la standardizzazione di strumenti in grado di misurare tale complessità in maniera psicometricamente corretta, cioè di strumenti accettabili anche per soggetti di basso livello socio-culturale (quali sono molti utenti dei servizi psichiatrici pubblici), sensibili, validi ed affidabili. Questi strumenti dovranno essere il più possibile utilizzati «sul campo». Diffondere a livello di base gli strumenti per la misurazione di parametri qualitativi di esito, al fine di consentire un monitoraggio di routine dell'intervento attuato dai servizi psichiatrici territoriali, è una delle principali sfide che attendono la psichiatria sociale ed epidemiologica nel prossimo decennio.

\section{BIBLIOGRAFIA}

Attkisson C., Cook J., Karno M., Lehman A. et al. (1992). Clinical services research. Schizophrenia Bullettin 18, 4, 627-668.

Brugha T. S. (1990). Social networks and support. Current Opinion in Psychiatry 3, 264-268.

de Jong A., Giel R., Soloff C. \& Wiersma D. (1986). Relation- ship between symptomatology and social disability. Social Psychiatry 21, 200-214.

De Salvia D. \& Tansella M. (a cura di) (1992a). Monitoraggio e Valutazione dei Servizi in 5 aree di Registro. Uno Studio Epidemiologico Comparativo (Prima parte). Epidemiologia $e$ Psichiatria Sociale 1, 15-60.

De Salvia D. \& Tansella M. (a cura di) (1992b). Monitoraggio e Valutazione dei Servizi in 5 Aree di Registro. Uno Studio Epidemiologico Comparativo (Seconda parte). Epidemiologia e Psichiatria Sociale 2, 83-148.

Fadden G., Bebbington F. \& Kuipers L. (1987). The burden of care: the impact of functional psychiatric illness on the patient's family. British Journal of Psychiatry 150, 273-285.

ten Horn G. H. M. M., Giel R., Gulbinat W. H., \& Henderson J. H. (1986). Psychiatric Case Registers in Public Health. A World-Wide Inventory 1960-1985. Elsevier: Amsterdam.

Lehman A. F. \& Burns B. J. (1990). Severe mental illness in the community: quality of life assessment. In Quality of Life Assessment in Clinical Trials (ed. B. Spilker). Raven Press: New York.

Mayer J. \& Rosenblatt A. (1974). Clash in perspective between mental patients and staff. American Journal of Orthopsychiatry 44, 432-441.

Mignolli G., Faccincani C. \& Platt S. (1991). Psychopathology and social performance in a cohort of patients with schizophrenic psychoses. A seven year follow-up study. In Communitybased Psychiatry: Long Term Patterns of Care in South-Verona (ed. M. Tansella). Psychological Medicine Monograph Supplement 19. Cambridge University Press: Cambridge.

Ruggeri M. (1991). Le ricerche sulla soddisfazione dei pazienti e dei loro familiari nei confronti dei Servizi Psichiatrici. Quaderni Italiani di Psichiatria 2, 89-124.

'Ruggeri M. \& Dall'Agnola R. (1993). The development and use of the Verona Expectations for Care Scale (VECS) and the Verona Service Satisfaction Scale (VSSS) for measuring expectations and satisfaction with community-based psychiatric services in patients, relatives and professionals. Psychological Medicine, 23, 511-523.

Ruggeri M., Dall'Agnola R., Agostini C. \& Bisoffi G. (1993). Acceptability, sensitivity and content validity of VECS and VSSS in measuring expectations and satisfaction with communitybased psychiatric services in patients and their relatives (inviato per la pubblicazione).

Tansella M. (1989). Evaluating community psychiatric services. In The Scope of Epidemiological Psychiatry (ed. P. Williams, G. Wilkinson and K. Kawnsley), pp. 386-403. Routledge: London.

Thompson C. (ed.) (1989). The Instruments of Psychiatric Research. John Wiley \& Sons: New York.

Thornicroft G., Brewin C. \& Wing J. (ed.) (1992). Measuring Mental Health Needs. Gaskell, The Royal College of Psychiatrists: London.

Wing J. K. (1990). Meeting the needs of people with psychiatric disorders. Social Psychiatry and Psychiatric Epidemiology 25, 2-8.

Wing J. K. \& Fryers T. (1976). Psychiatric Services in Camberwell and Salford. Statistics from the Camberwell and Salford Psychiatric Registers, 1964-1974. Institute of Psychiatry: London. 
ARTE E PSICHE.

Fenomenologia della creatività da Leonardo a Magritte

di Ellen Handler Spitz

Un libro di 212 pagine, con otto tavole a colori fuori testo.

Lire 45.000

Come arrivare ad un approccio integrato e interdisciplinare alla critica d'arte?

Candidata speciale al Centre for Psychoanalytic Training and Research della Columbia University, Ellen Handler Spitz ci propone un'analisi stimolante dei tre principali approcci psicoanalitici all'arte, considerata nelle sue più diverse espressioni: pittura, scultura, letteratura, musica, danza.

Una lettura da consigliare a tutti coloro che si preoccupano di sviluppare il dialogo interdisciplinare tra psicoanalisi, estetica e storia della critica.

L'edizione italiana è presentata da Maurizio Bonicatti, professore ordinario presso la Facoltà di Lettere e Filosofia di Roma "La Sapienza"; didatta presso l'Associazione Ricerche in Psicologia Applicata - Comitato di consulenza scientifica.

Hanno detto del libro:

"Nessuno, tra quanti coinvolti nello studio psicoanalitico dell'arte, potrà non considerare quest'opera; come nessuno, d'altro canto, tra i cultori dell'arte psicoanalitica, potrà ignorarla".

Journal of the American Psychoanalytic Association

"Raccomandiamo la lettura del libro a tutti coloro che credono che le molteplici applicazioni del pensiero analitico possano contribuire a migliorare le nostre capacità di giudizio".

Journal of the American Academy of Psychoanalysis

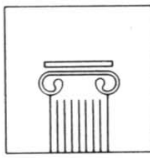

Il Pensiero Scientifico Editore

via Bradano 3/c - 00199 Roma

Tel. (06) 86207158/59/68/69 